\title{
ON THE DESIGN AND REALIZATION OF ADAPTIVE EQUALIZATION FOR MOBILE COMMUNICATION
}

\author{
J. van Bussel ${ }^{1}$ \\ C.J.E. van Heerde $e^{1,2}$ \\ C.H. Slump ${ }^{1}$ \\ M.J. Bentum 1 \\ ${ }^{1}$ University of Twente, Dept. of Electrical Engineering, P.O. Box 217, Enschede, the Netherlands \\ ${ }^{2}$ Ericsson Business Mobile Networks, P.O. Box 645, Enschede, the Netherlands
}

\begin{abstract}
In this paper the reduction of InterSymbol Interference (ISI) in mobile communication channels is addressed. A well known technique for ISI reduction is adaptive equalization in which the time-varying characteristics of the mobile channel is tracked by an adaptive algorithm. We compare different adaptive strategies and equalizer structures with respect to overall system performance in terms of computational complexity, numerical stability, finite wordlength effects, size and power consumption required for VLSI implementation.

The adaptation strategies addressed are linear forward, decision feedback equalization. The selected algorithms are simulated in the Signal Processing Worksystem (SPW) tool complying to the Global System Mobile (GSM) standard and the Digital Enhanced Cordless Telecommunications (DECT) standard. The performance of the pertinent algorithm is expressed as the Bit Error Rate (BER) versus the Signal-to-Noise Ratio (SNR). Robustness and stability, the effects of finite wordlength and expected power consumption are presented as well.
\end{abstract}

\section{INTRODUCTION}

The current generation of mobile communication uses digital modulation techniques to transmit speech and data. The analog transmission channels deliver distorted versions of the original signal, so called InterSymbol Interference (ISI). To recover the data signal, equalization techniques are often used in the receiver. In Figure 1 several types [1] are shown.

We evaluate these techniques with respect to the following aspects: BER versus SNR, power consumption, computational complexity and the numerical stability. The Viterbi algorithm is a maximum likelihood estimator which is commonly used in mobile communications. The disadvantage of this Maximum Likelihood Sequence Estimation (MLSE) algorithm is the high computational complexity when the impulse response to be equalized spans several symbols [1][2][3]. We therefore focus on the Decision Feedback Equalizer (DFE) which is also suitable for use in mobile communications. The DFE can be equipped with different types of adaptation techniques which are numerically stable and has a low computational complexity. We investigate the implications of fixed point arithmetic on behalf of VLSI implementation. The finite length realization of these

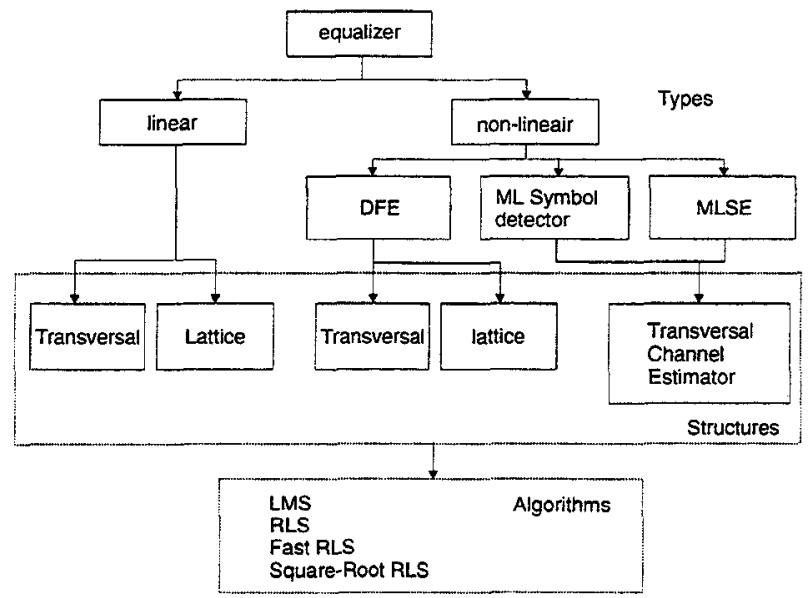

Figure 1. Equalization of transmission channels [1].

algorithms in VLSI determines the numerical stability.

\section{CHANNEL MODELING}

Figure 2 shows the transmission model which is used for testing the different types of equalizers with the several adaptation algorithms. There are different digital communication systems such as: the DECT, the GSM and the American standard IS-54 [4]. The GSM standard has been used as a test environment in SPW simulations. However, the selected equalizers and the pertinent adaptation algorithms are also suitable for use in a DECT or IS-54 standard.

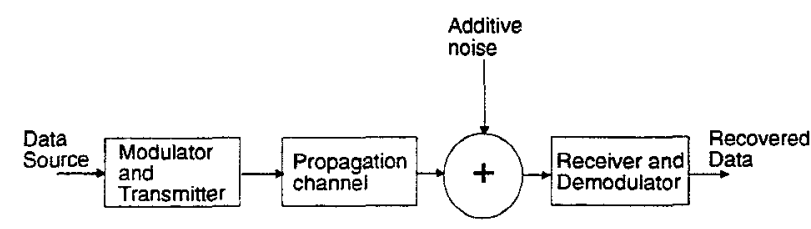

Figure 2. Transmission model.

In order to test and evaluate the various methods of equalization, an appropriate channel model is needed. The received signal is a weighted linear combination of the transmitted signal and therefore a linear equalizer can be used to combat the ISI. To model the channel, a tapped-delay-line 
(TDL) with time-varying tap-weights is used. These tapweights are determined by a Gaussian random process with a power spectral density varying with the Doppler spectrum. The delays and average tap-weights used here are taken from the ETSI recommendation GSM 5.05, given by

TU-50 typical case for urban area at $50(\mathrm{~km} / \mathrm{h})$ tap-weights $=0.4,0.5,1.0,0.63,0.5,0.32,0.2,0.32$, $0.25,0.13,0.08,0.1$

delay $=0,0.2,0.4,0.6,0.8,1.2,1.4,1.8,2.4$ $3.0,3.2,5.0(\mu \mathrm{s})$

HT-100 typical case for hilly terrain at $100(\mathrm{~km} / \mathrm{h})$

tap-weights $=0.1,0.16,0.25,0.4,1.0,1.0,0.4,0.16$, $0.13,0.1,0.06,0.04$

$$
\text { delay }=0.0,0.2,0.4,0.6,0.8,2.0,2.4,15.0 \text {, }
$$
$15.2,15.8,17.2,20.0(\mu \mathrm{s})$

The hilly terrain has a larger delay time than the urban area. Because of this larger delay time, an investigation is done on using a DFE instead of an MLSE equalizer in this situation.

\section{EQUALTZERS}

The most commonly used equalizer is the Linear Equalizer. The disadvantage of using this type of equalizer is the sensitivity to spectral nulls in the frequency response of the radio channel. It enhances the noise in these points. To avoid this effect a non-linear Decision Feedback Equalizer (DFE) structure can be used. This equalizer consists of two parts, i.e. a feedbackward and a feedforward part.

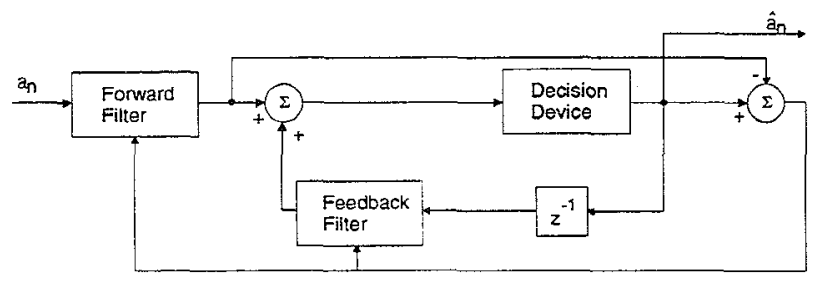

Figure 3. Decision Feedbackward Equalizer (DFE)

The feedbackward part of the DFE is used to cancel the postcursor of the impulse response. Because the feedbackward part cannot remove the precursor of the impulse response, the DFE has a feedforward part which handles this part. By combining both functions they perform, in a sense, complementary functions. In Figure 3 and 4, two types of implementations of the Decision Feedback Equalizers are given [1][5].

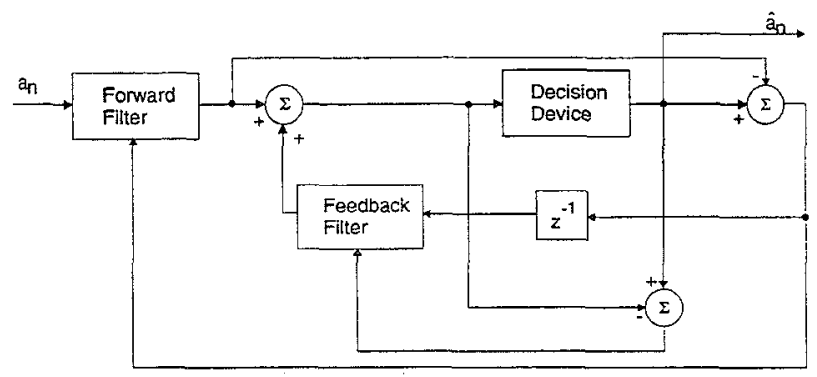

Figure 4. Predictive DFE

\section{SIMULATION RESULTS}

The modulator, the demodulator and a channel of the GSM standard are implemented for testing the equalizers and their adaptation algorithms. GSM uses GMSK modulation and has a carrier frequency of about $900 \mathrm{MHz}$ and a symbol rate of $270 \mathrm{kbit} / \mathrm{s}$. The used equalizers has to eliminate a maximum of $16(\mu \mathrm{s})$ ISI in the received signal (GSM recommendation 5.05). These are with a symbol time of 3.7 $(\mu \mathrm{s})$ about 4 to 5 symbols. The forward equalizer of the DFE is fractionally spaced $\left(\frac{T}{2}\right)$. This makes the equalizer independent of the sampling clock phase (clock recovery). It is assumed that the length of the equalizer spans the total introduced ISI in the concerned communication channel, so that the number of forward taps is about 8 to 10 taps. The backward equalizer of the DFE is T-spaced and the number of taps is varied between 2 and 4 [1]. The number of taps depends not only on the symbol time but also on the channel characteristics. Figures 5 to 8 show the performance of several adaptation algorithms for the estimation of the channel characteristics. All simulations are made with a finite convergence time (10,000 samples). The performance of the equalizer is expressed as the BER versus the SNR.

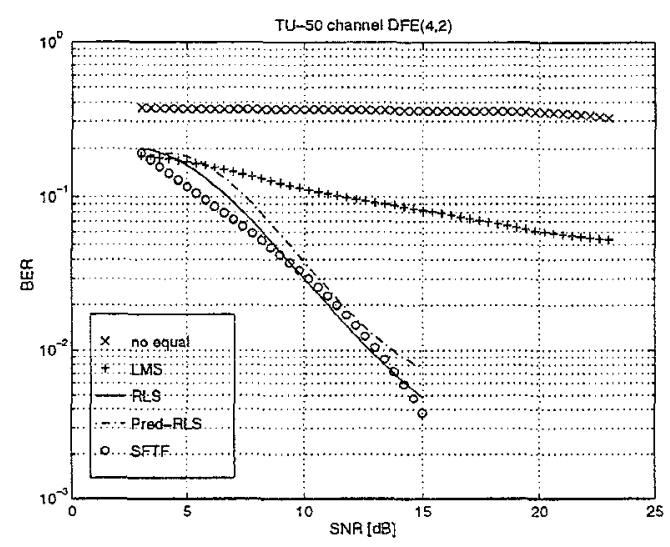

Figure 5. TU-50 channel $\operatorname{DFE}(4,2)$

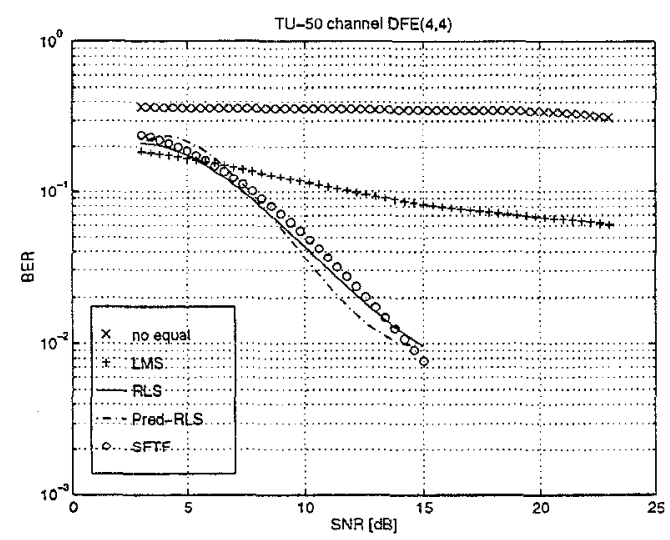

Figure 6. TU.50 channel $\operatorname{DFE}(4,4)$

These Figures show that the SFTF [6][7] and the RLS adaptation algorithms have the best performance. Because 


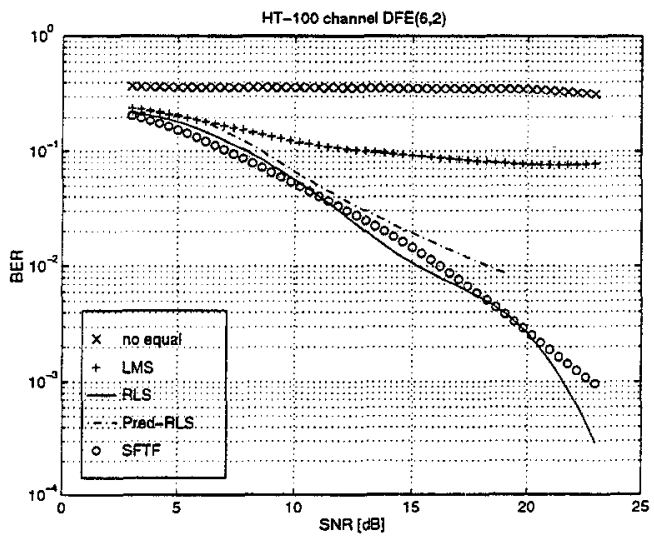

Figure 7. HT-100 channel DFE(6,2)

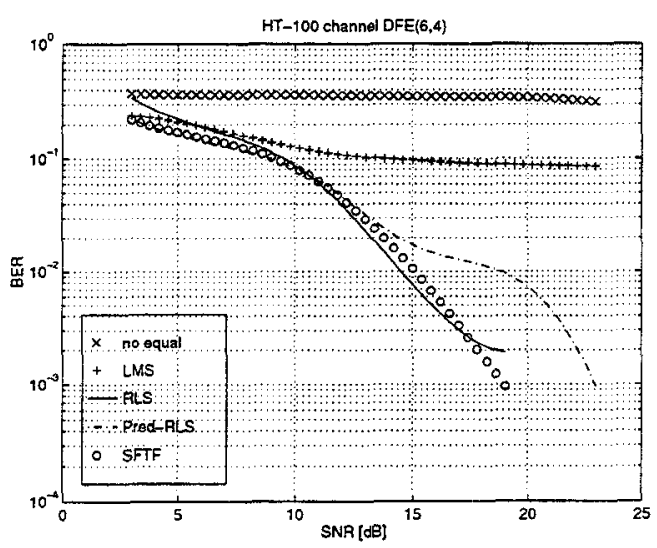

Figure 8. HT-100 channel DFE(6,4)

the computational complexity of the SFTF algorithm is lower, this algorithm is of main interest. This algorithm is a stabilized version of the Fast Transversal Filter algorithm. The simulated adaptation algorithms are programmed in floating point notation, however in mobile communications the power consumption and numerical stability are important criteria. A low computational complexity implies a low power consumption, in the sense that less computations (e.g. multiplications, divisions) result in less power dissipation.

The computational complexities [1] of several adaptation algorithms are depicted in Figure 9. The forward and backward taps are assumed to be equal.

Instead of using equalizers based on linear adaptive filter theory, with weights being adapted according to gradient algorithms, neural networks are an option for use in mobile communications. In the simulation of the linear adaptive filters it has been found that the implemented equalizers are sensitive to noise in the received signal.

\section{FINITE PRECISION EFFECTS}

The computational complexity can also be reduced by implementing the equalizer in fixed point arithmetic. The interest lies in finding the minimal wordlength at which the criteria for effective equalization are sufficiently satisfied.

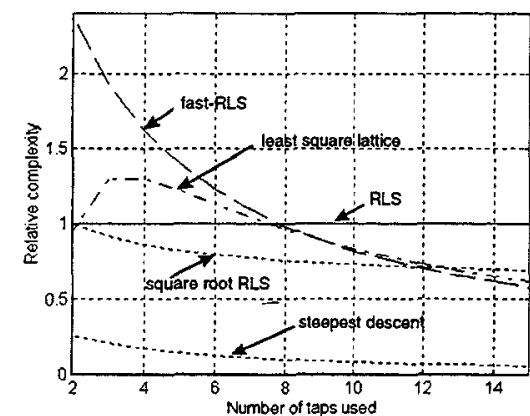

Figure 9. Relative computational complexity versus the total number of taps

The adaptive algorithm is to be implemented in VLSI, due to the demand for low-power and compact mobile units. Due to the finite precision of the fixed point implementation of the algorithm, numerical errors may occur. Algorithms such as the FTF [8][9] and the GFTF [10] suffer from roundoff error propagation, which may result in numerical instability. This instability can be prevented by the use of the Stabilized FTF (SFTF) and the Modified Stable GFTF (MSGFTF) algorithm [11]. Both algorithms have indeed appeared to remain stable in fixed point arithmetic. However, the MSGFTF algorithm has a deviation from the exact least-squares solution, which becomes more noticeable with smaller wordlengths.

A -simplified- test environment for the linear equalizers is an environment in which a QPSK signal is distorted by a multipath channel by Rummler [12].

The aim of this research is to find the optimal (minimal) wordlength. It is assumed that all parameters and signals in the algorithm have the same wordlength. Signals or parameters with similar features (such as amplitude range, desired accuracy) can be combined in a group (or class). This results in groups of signals or parameters that have equal wordlengths, but may differ in the number of fraction bits (integer bits). This may lead to a lower overall wordlength.

The algorithms in fixed point arithmetic have first been tested in two's complement with a default wordlength of 64 bits, of which 31 bits are integer bits, denoted as: $\langle 64,31, t\rangle$. The simulation results were corresponding to the floating point simulations. However, the difference between the algorithms become evident if the overall wordlength is reduced. The finite precision effects become more noticeable with the reduction of the wordlength. To verify which algorithm is most resistant to these unwanted effects, the fixed point attributes have been reduced to $\langle 45,21, t\rangle$. This value was found to be the minimum overall wordlength for which the SFTF algorithm does not suffer from overflow. Following this, the GFTF and the MSGFTF algorithm have been simulated with these attributes. See Figure 10. It is clear from these simulation results, that the GFTF algorithm suffers greatly from fixed point effects. The MSGFTF algorithm remains stable, but has a larger resulting error.

An effective implementation of the adaptive algorithm 

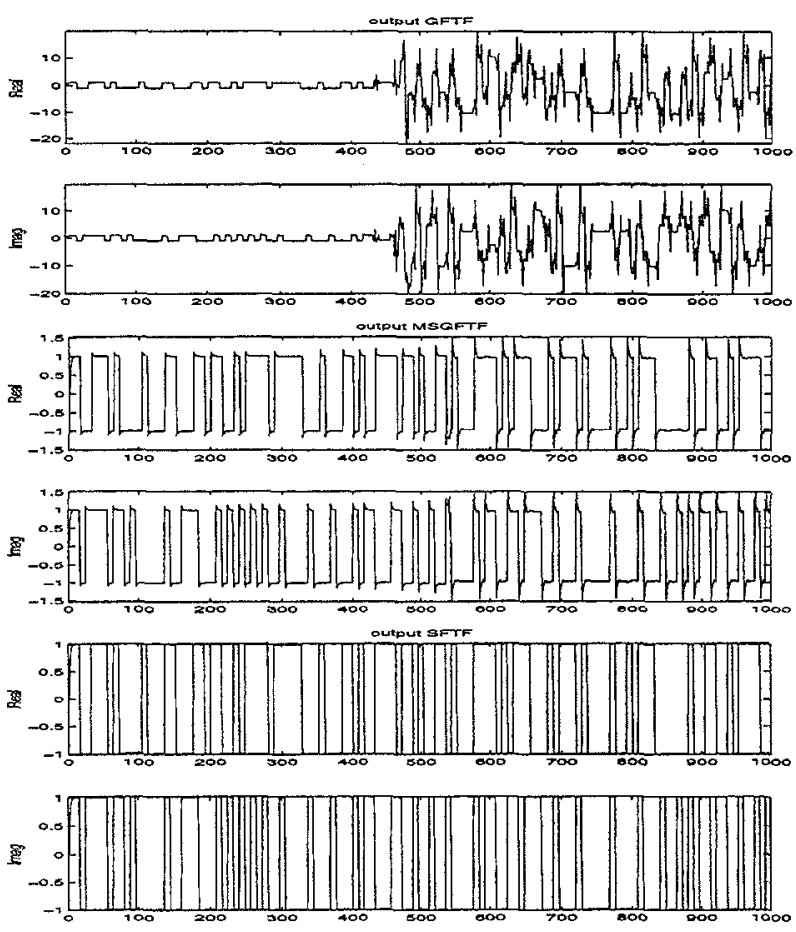

Figure 10. Simulation results $(\langle 45,21, t\rangle)$

in fixed point arithmetic requires exact knowledge of the internal structure of the algorithm. By describing the algorithm for adaptive equalization in a state-space form, efficient scaling methods can be implemented to achieve a lower wordlength. The scaling factors are determined a priori, thus increasing the computational complexity by a fraction.

Proper scaling of state components, coefficients and input leads to an implementation also known as block-floatingpoint implementation [13][14]. This type of implementation results in a larger dynamic range than can normally be achieved with fixed point implementation.

\section{CONCLUSIONS AND RECOMMENDATIONS}

In mobile communications, high performance and a low power consumption are important features. The simulations show the performance of various adaptation algorithms with two channel models. The used adaptation algorithms are first implemented in floating point arithmetic. For implementation in VLSI the algorithms are converted into fixed point arithmetic. The GFTF algorithm becomes unstable due to numerical error propagation, whereas the SFTF algorithm has been found to be the 'best' FTF-based algorithm. The performance of the SFTF depends on the used number of taps and channel type. Simulations show that increasing the number of feedback taps decreases the performance. This is due to the influence the 'wrong' decisions have on the following decisions.

It has also been found through simulations that for smaller values than the attributes $\langle 45,21, t\rangle$ (i.e. 45 bits wordlength, of which 21 integer bits, in two's complement), all three FTF-based algorithms (GFTF, MSGFTF, SFTF) become unstable because of overflow.

By properly scaling internal parameters of the adaptation algorithms, the inherent roundoff error can be reduced. Moreover, it is possible to further reduce the wordlength with this scaling method. Further research is currently done on this issue.

\section{REFERENCES}

[1] J. Proakis, "Adaptive equalization for TDMA digital mobile radio," IEEE Trans. on Vehicular Technology, vol. 40, pp. 333-341, May 1991.

[2] S. Chen, S. McLaughlin, and P. M. Grant, "Adaptive Bayesian Decision Feedback Equalizer for dispersive mobile radio channels," IEEE Trans. on Communications Systems, vol. 43, pp. 1937-1945, May 1995.

[3] J.Tellado-Mourelo, E. Wesel, and J.M.Cioffi, "Adaptive DFE for GMSK in indoor radio channels," IEEE Trans. on Communications Systems, vol. 14, pp. 492501, April 1996.

[4] D. Goodman, "Second generation wireless information networks," IEEE Trans. on vehicular technology, vol. 40, pp. 366-374, May 1991.

[5] S. Benedetto, E. Biglieri, and V. Castellani, Digital Transmission Theory. Prentice-hall, 1987.

[6] D. T. Slock and T. Kailath, "Numerically stable fast transversal filters for Recursive Least Square adaptive filtering," IEEE Transactions on Signal Processing, vol. 39, pp. 92-114, January 1991.

[7] N. Kalouptsidis and S. Theodoridis, Adaptive system identification and signal processing algorithms. Prentice Hall, 1993.

[8] D. T. Slock and T. Kailath, "Fast, Recursive-LeastSquare transversal filters for adaptive filtering," Transactions on Accoustic, Speech and Signal Processing, vol. 32, pp. 304-337, April 1984.

[9] S. Haykin, Adoptive Filter Theory. Prentice Hall, 1991.

[10] S. T. Alexander, Adaptive Signal Processing - Theory and Applications. Springer-Verlag, 1986.

[11] Z. Ren and H. Schütze, "A stabilized fast transversal filters algorithm for Recursive Least Squares adaptive filtering," Signal Processing, vol. 39, pp. 235-246, April 1994.

[12] Alta-Group, Signal Processing WorkSystem manuals. Cadence Design Systerns Inc., 1995. Product Number : SPW8012.

[13] D. Williamson, Digital Control and Implementation. Prentice Hall, 1991.

[14] K. Kalliojärvi and J. Astola, "Roundoff errors in blockHoating-point systems," IEEE Transactions on signal processing, vol. 44, pp. 783-790, April 1996. 